\title{
DUBAI 3D TEXTUERD MESH USING HIGH QUALITY RESOLUTION VERTICAL/OBLIQUE AERIAL IMAGERY
}

\author{
Adib Tayeb Madani ${ }^{\text {a }}$, Abdullateef Ziad Ahmad ${ }^{\mathrm{a}^{*}}$, Lueken Christoph ${ }^{\mathrm{a}}$, Zamzam Hammadi ${ }^{\text {a }}$, Manal Abdullah Sabeal ${ }^{\mathrm{a}}$ \\ ${ }^{a}$ Geographic Information System Department, Dubai Municipality, Dubai, United Arab Emirates - \\ (atmadani, azahmed, lchristoph, masabeal)@dm.gov.ae
}

\section{Commission III, WG III/2}

KEY WORDS: Oblique, Texturing, 3D, Dense Matching, Mesh, Dubai Municipality, DSM.

\begin{abstract}
:
Providing high quality 3D data with reasonable quality and cost were always essential, affording the core data and foundation for developing an information-based decision-making tool of urban environments with the capability of providing decision makers, stakeholders, professionals, and public users with 3D views and 3D analysis tools of spatial information that enables real-world views. Helps and assist in improving users' orientation and also increase their efficiency in performing their tasks related to city planning, Inspection, infrastructures, roads, and cadastre management. In this paper, the capability of multi-view Vexcel UltraCam Osprey camera images is examined to provide a 3D model of building façades using an efficient image-based modeling workflow adopted by commercial software's. The main steps of this work include: Specification, point cloud generation, and 3D modeling. After improving the initial values of interior and exterior parameters at first step, an efficient image matching technique such as Semi Global Matching (SGM) is applied on the images to generate point cloud. Then, a mesh model of points is calculated using and refined to obtain an accurate model of buildings. Finally, a texture is assigned to mesh in order to create a realistic 3D model. The resulting model has provided enough LoD2 details of the building based on visual assessment. The objective of this paper is neither comparing nor promoting a specific technique over the other and does not mean to promote a sensor-based system over another systems or mechanism presented in existing or previous paper. The idea is to share experience.
\end{abstract}

\section{INTRODUCTION}

Oblique airborne photogrammetry is rapidly maturing and becoming almost standard in many mapping applications especially for 3D City modeling thanks to the development of airborne digital multi-camera systems. There are various applications of 3D building modeling such as urban mapping, virtual reality, 3D visualization, and analyzing.

With the current significant improvements on camera sensors and multi-ray Photogrammetric applications, and tools, Software's starts affording the required efficient tools that are capable of generating dense cloud points from sensor-based highly overlapped Nadir imagery in order to produce and reconstruct 3D Mesh for the 3D buildings and attach the best facade textures for those objects Automatically by using a preacquired Oblique imagery that best fit the 3D object.

A knowledge based modeling can be established to reconstruct the building façade using both geometric and semantic features. For this, the fusion between sensor based imagery point clouds and close range images helps to reduce the cost comparing to using traditional fusion between Airborne and terrestrial Lidar technologies for the generation of LoD2 building 3D Models (with of course keeping in mind the power and importance of Terrestrial Lidar as input for generating LoD3 comprehensive city Model over the usage of sensor based imagery) and also Improve the completeness and adaptability of modeling.
As known, cost and quality and accuracy prior to adopt any Approach for extracting and reconstruction 3D objects will always be an important key factor that needs to be considered and assessed carefully when executing any approach especially when data are acquired for Analysis and Visualization? imagebased modeling approach were chosen due to the fact that Dubai Municipality has a rich collection of high resolution Nadir based aerial imagery with decent overlap that facilitate the generation of enough dense cloud points that will be the source for producing a sufficient and accurate enough 3D Mesh .

With the enhancements and development of camera systems Vexcel Ultracam osprey was selected to acquire high resolution vertical/Oblique Imagery Will result in a unified aerial triangulation for both Aerial and Oblique Imagery in order to ensure superior accuracy in imagery orientation which supposed to guide to more accurate and reliable prediction of textured surfaces of the 3D Objects and It also provides a superior dataset for the extraction of highly detailed DSM.

In this paper we are explaining and sharing the knowledge and experience in using several semiautomatic methods adopted by commercial software's like (Bentley Acute3D, SURE, Skyline) for reconstructing 3D textured buildings from multi view (nadir/oblique) images. 


\section{PRE-FLYING PLANNING}

Oblique imagery provides more deep and descriptive information about urban areas, in order to be able to produce detailed mesh, dense enough cloud points need to be generated and proper oblique imagery for the AOI has to be generated; therefore the following specification had been applied and ensure:

- Choose the highest allowed and achievable GSD for the AOI which as result ranges between $(10-20 \mathrm{~cm})$ in average depend on the flying altitude allowed by local official regulators

- Increase the forward overlap of strips from $60 \%$ to $80 \%$.

- Increase the side overlap from $40 \%$ to $80 \%$.

- Nadir/Oblique imagery to be acquired in the same flight mission for more unified AT process and uniform results.

For best quality and to avoid high and long shadows the azimuth Sun angle during image acquisition were not less than 30 degrees cloud or cloud shadow does not exceed $5 \%$ of the area of anyone photograph

With the above mentioned specification the output imagery were sufficient to process and produce $3 \mathrm{D}$ building model for the desired AOI.

\section{CAMERA SYSTEM}

It was always in mind that the quality of the automated dense matching process depends significantly on the camera and the structure of the terrain. Geometric stability and radiometric dynamic of the camera have a direct impact on the matching results.

Therefore and in order to use a combined solution for Vertical and Oblique Aerial Photography the class leading Microsoft UltraCam Osprey Oblique Aerial Sensor was selected due to the fact that it houses two cameras in one photogrammetric grade housing using cutting edge technology to collect photogrammetry-grade Nadir images (PAN, RGB, and NIR) and Oblique images (RGB) simultaneously.

This system combines high performing photogrammetric nadir camera with oblique capture capability to deliver superior image quality and performance and it's ultimately suited for urban mapping and 3D City Modeling.

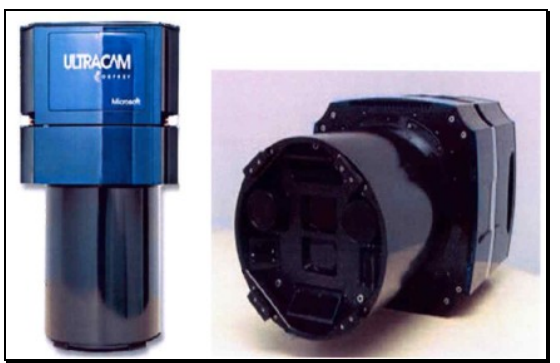

Figure 1. ULTRACAM Osprey
The photogrammetric nadir part of the camera features a PAN Cross pixel of 11,674 across the flight strip, RGB color cone and NIR cone (Pan-Sharpen ratio 1:2).

This in turns makes the nadir subsystem a high performing digital Photogrammetric camera that delivers sub-pixel accuracy because both Nadir and Oblique imagery was acquired from the same camera at the same time on the same flight mission by the same aircraft is a unique approach which offers the following benefits:

- The vertical and oblique images will be consistent taking in consideration that it is a Metric Camera, which means that accuracy needed will be obtained.

- All tasks (AT, DSM production...etc) will be consistent with each other leading to fewer errors to be manually rectified.

The overlap is based on the nadir looking camera with $80 \%$ Forward overlap and $80 \%$ side lap. The oblique sensors will In turn have a larger overlap due to their incidence angle.

In this paper, the workflow followed to reconstruct a 3D model of buildings and façades textures from imagery captured using UltraCam Osprey images (nadir/oblique) is presented and shared (Figure 1). The main steps of this workflow include:

- $\quad \mathrm{AT}$ and Block Bundle Adjustment

- Point cloud and DSM generation,

- $\quad 3 \mathrm{D}$ modeling and Texturing.

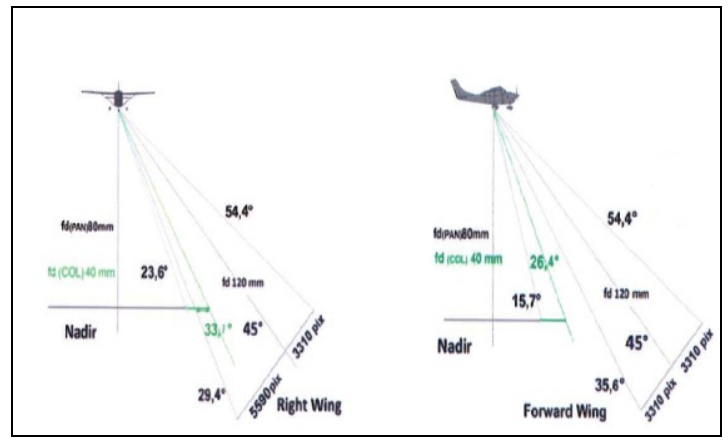

Figure 2.

\section{IMAGE PROCESSING WORKFLOW}

In this paper, the workflow followed to reconstruct a 3D model of buildings and façades textures from imagery captured using UltraCam Osprey images (nadir/oblique) is presented and shared (Figure 1). The main steps of this workflow include:

- $\quad$ AT and Block Bundle Adjustment

- Point cloud and DSM generation

- $\quad 3 \mathrm{D}$ modeling and Texturing 


\subsection{AT \& Block Bundle Adjustment Process}

GPS and IMU assisted AT used, it provides superior control of the final AT. It provides for accuracies in the adjusted object coordinates better than 0.4 pixels in $\mathrm{x}, \mathrm{y}$ and better than one pixel in $\mathrm{Z}$. The following will outline the special care used to avoid certain pitfalls in GPS and IMU assisted AT.

Lever arms for GPS and IMU are surveyed with terrestrial methods using over determination to exclude gross errors of The lever arms. Lever arms are documented accurate up to 10 $\mathrm{mm}$, which are sufficient to produce highly consistent GPS and IMU data referenced to the projection center without any residual errors from the lever arms.

In order to triangulate the oblique imagery procedures for AT of vertical images were adopted.

The final adjustment were a combined adjustment of both vertical and oblique imagery, a task that were handled by BINGO software

The results were having better orientations which are completely uniform over the entire data set of both vertical and oblique images.

The tie point collection for the oblique images performed using SOCET SET v. 5.6.0; a block is set up in SOCET SET that was configured to use both the vertical and oblique images.

SOCET SET is remarkable in its adaptation for a large number of sensor models, including oblique imagery up to a mounting angle of about 45 degrees. In this way, contiguous points are extracted across both the vertical and oblique images, ensuring that both data sets are tied together.

3D coordinates were calculated using some corresponding features of images, ground control points, Dubai Virtual Reference stations (DVRS) in addition to initial parameters in an Automatic Aerial Triangulation (AAT) and Bundle Block Adjustment (BBA) solution.

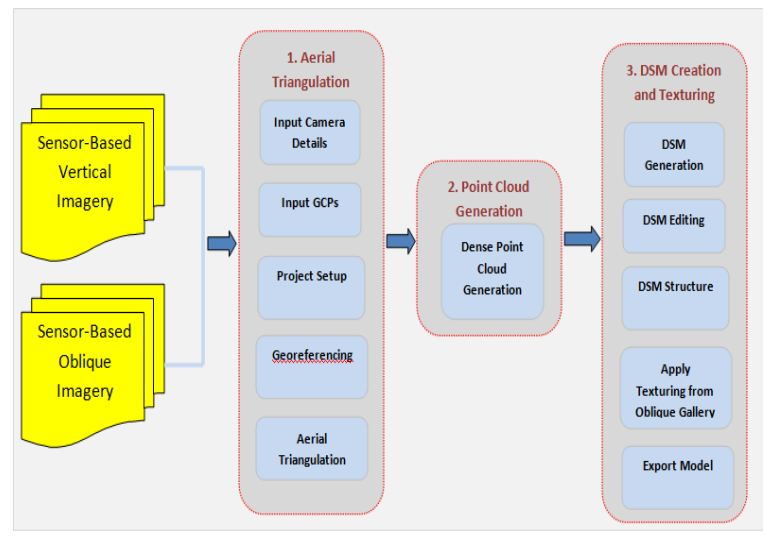

Figure 3. Processing Workflow

\subsection{DSM Generation}

After Calculating the AT parameters, the range values are calculated from image information of the corresponding pixels in all of images. The DSM was produced by SURE software from frame.

SURE offers several benefits, chiefly the very accurate results of multi-image matching and advanced filtering of outliers. The noise level produced by SURE is very minimal and below the required threshold, as documented in several results and articles detailing the software. Especially using an image dataset with $80 / 80$ overlap and oblique images at the same time, the edges of buildings become very clear and detailed, as well as the multiray triangulation of final point cloud coordinates are of superior accuracy.

Upon extraction of the raw point cloud, which has a density of up to one point per pixel, a DSM is calculated internally in the software. The DSM calculation has filtering algorithm applied to the DSM to remove any outlier points, however what was noticed with highly overlapping imagery is that significant outliers even in the point cloud is at a very minimum.

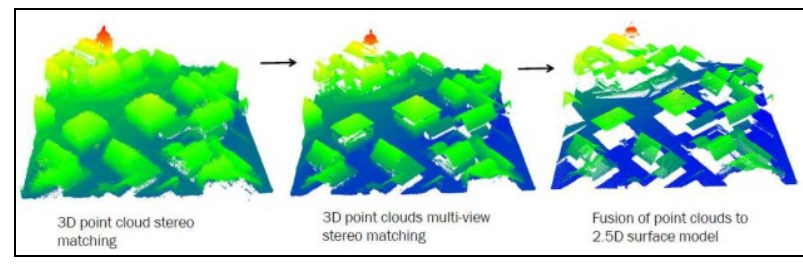

Figure 4. DSM Filtering

The extracted DSM was verified by using LiDAR tools to check the consistency of the extracted DSM, as well as a visual inspection.

The accuracy of the DSM performed by comparing the derived DSM to the triangulated object points from the AT . Due to the fact that the object points from the AT are part of the DSM, they were suitable for the task of checking the quality of the DSM generation.

\subsection{DSM Texturing}

The DSM based 3D modeling step comprises mesh generation and refinement, and texture mapping.

The DSM-based 3D city model is generated using the high quality Digital Surface Model (DSM) created using the professional photogrammetry software SURE, which applies Semi-Global Matching algorithms and therefore fulfils the Requirement of a high quality and accurate surface model

This DSM used in its native format in SURE, which ensures the highest possible coherence between all involved data sets.

SURE allows the automatic texturing of the generated DSM using the high-resolution aerial and oblique images and provide a photo-realistic DSM-based 3D city model for the whole AOI

The accuracy of generated mesh depends on the number of triangulations which are defined by deviation error. When the deviation error decreases, the number of points increases. Using 
The high values of deviation error, the level of detail is low and the resulting surface is roughly defined. Using the small values of deviation error, the resulting surface contains many spikes. So, the best accuracy of mesh is achieved by finding an optimum value of deviation error.

Finally, in multi-texturing step, the intensity information from multiple pixels of same triangle is combined to produce a unique texture. For this, based on the known internal and external camera parameters of original images, the texture is assigned (from the Oblique data source) to each triangle in 3D model, so the misalignment between textures from different sources could be very small.

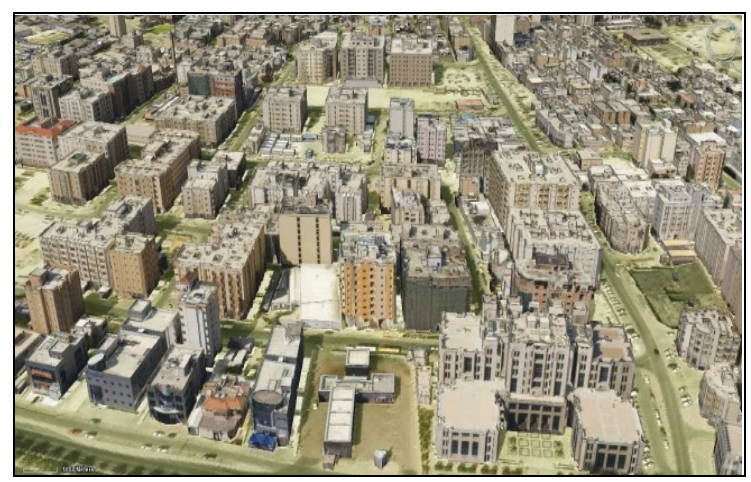

Figure 5. Textured Model Sample 1

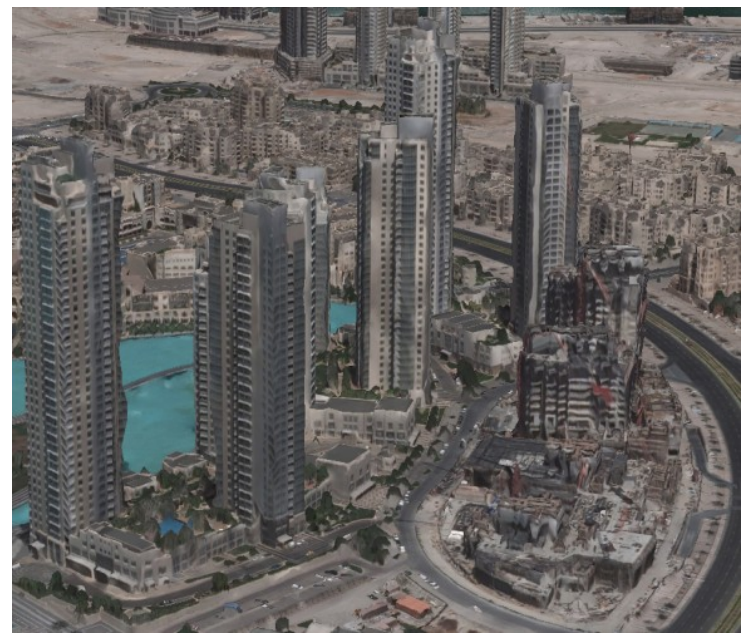

Figure 6. Textured Model Sample 2

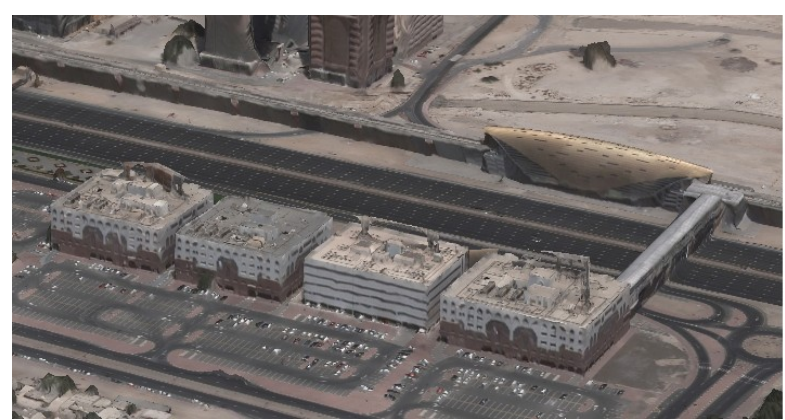

Figure 7. Textured Model Sample 3

\section{CONCLUSION}

There are many measurement systems or techniques to generate point cloud for $3 \mathrm{D}$ modeling such as $3 \mathrm{D}$ scanners, digitizers, CMM, laser trackers, laser planes (laser triangulation), time-offlight lasers (laser-grammetry), photogrammetry or stereophotogrammetry. The efficient method for 3D modeling is to generate a mesh from point cloud, so the details can be visible from different viewpoints. In this paper, Dubai Municipality experience in using sensor based images (oblique/Nadir) for the whole emirate is investigated for modeling of urban buildings based on three steps: AT, point cloud and DSM generation, and $3 \mathrm{D}$ texturing and modeling. In this workflow, the interaction of user is limited to initialize some parameters and there is no need to additional dataset such as primary CAD models. Also, the best result of modeling depends on the quality of the original point cloud and user's expectations. Moreover, the large memory requirement due to very dense data is the main problem and critical issue in this work.

The output result of this project has been shown that both nadir and oblique views can be used for mapping textures on virtual models of an urban scene without any loss of quality.

\section{ACKNOWLEDGEMENT}

It's important to thank all who participated and supported this project from the internal Dubai municipality teams and external vendors especially (Mr. Abdulhakim Malik, Mrs Eman Humaid, Mrs Maryam Tayer, Mrs Ayda Shukralla, Mr Mads Maaløe Bach , Mr Michael Schultz Rasmussen)

\section{REFERENCES}

Hirschmuller, H., 2008. Stereo processing by semiglobal matching and mutual information. IEEE Transactions on Pattern Analysis and Machine Intelligence 30(2), pp. 328-341.

Lehmann, F. et al., 2011. MACS - Modular Airborne Camera System for generating photogrammetric high-resolution products. Photogrammetrie, Fernerkundung, Geoinformation. 6, pp. 423-434.

Stilla, U., Kolecki, J. and Hoegner, L., 2009. Texture mapping of 3D building models with oblique direct geo-referenced airborne IR image sequences. Archives of the ISPRS 38-1-47/W5.

D. Frommholz, M. Linkiewicz, H. Meissner, D. Dahlke, A. Poznanska . Extracting semantically annotation 3D building models textures from oblique aerial imagery. 